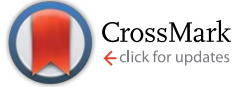

Cite this: J. Mater. Chem. A, 2015, 3, 1594

Received 20th October 2014

Accepted 24th November 2014

DOI: $10.1039 / \mathrm{c} 4 \mathrm{ta} 05618 \mathrm{c}$

www.rsc.org/MaterialsA

\section{Synthesis of mechanically reinforced silica aerogels via surface-initiated reversible addition- fragmentation chain transfer (RAFT) polymerization $\dagger$}

\author{
H. Maleki, ${ }^{\star}$ L. Durães and A. Portugal
}

Mechanically reinforced polymer-silica aerogels have been successfully prepared by using surface-initiated reversible addition-fragmentation chain transfer polymerization. With this approach, well-defined polystyrene (PSt) and poly(butyl acrylate) (PBA) with low polydispersities grew on the silica surface and improved the mechanical strength in relation to native aerogels. Moreover, it allowed establishing a structure-property relationship between the grafted polymer molecular weight and physical properties of the hybrid aerogels, thereby enabling the preparation of composites with tailored properties. The aerogel composites here obtained exhibited a low density of $0.13-0.17 \mathrm{~g} \mathrm{~cm}^{-3}$, high thermal insulation performance of $0.03-0.04 \mathrm{~W} \mathrm{~m}^{-1} \mathrm{~K}^{-1}$ and a high specific surface area of $350-780 \mathrm{~m}^{2} \mathrm{~g}^{-1}$, with approximately one order of magnitude improvement in the compression strength over the nonreinforced aerogels.

\section{Introduction}

The extraordinary properties of silica aerogels, such as low density, high specific surface area and extremely low thermal conductivity, make them attractive materials for numerous applications. Some of these applications involve the use of silica aerogels in Cerenkov radiation detectors of certain nuclear reactors, ${ }^{1}$ as collectors of hypervelocity particles in space, ${ }^{2,3}$ as thermal insulators for space vehicles ${ }^{4}$ and as low $k$-electric materials. ${ }^{5}$ However, it is complicated to handle and process native silica aerogels without making severe fracture on the material. Therefore, improving the mechanical properties of silica aerogels has been a great challenge to achieve a material that may fully retain their attractive properties for potential applications. Different strategies have been investigated to reinforce silica aerogels through enlarging the connection points between silica secondary particles in the aerogel backbone. ${ }^{6}$ In this regard, it is possible to carry out reinforcing before supercritical drying, via a liquid phase, ${ }^{7-9}$ or after supercritical drying, performing it through a gas phase..$^{10,11}$ The "aging" process, which is the reinforcing of silica in the neck region (through existing silica on the network), and compounding the silica wet gel surface with different polymer networks (epoxy, urethane, urea, styrene, etc.) are the liquid phase reinforcing approaches to

CIEPQPF, Department of Chemical Engineering, Faculty of Sciences and Technology, University of Coimbra, Rua Sílvio Lima, 3030-790 Coimbra, Portugal. E-mail: hajar@eq.uc.pt; Fax: +351 239798703; Tel: +351910156989

$\dagger$ Electronic supplementary information (ESI) available. See DOI: $10.1039 / \mathrm{c} 4 \mathrm{ta} 05618 \mathrm{c}$ improve silica aerogel's mechanical strength., ${ }^{\mathbf{8 , 1 2}}$ Almost all mechanical reinforcing strategies have led to some extent an undesirable increase in the bulk density and thermal conductivity, and a reduction of the surface area. ${ }^{7,13}$

In terms of polymer reinforcement, recent studies have shown that optimal mechanical reinforcement of aerogels could be achieved by grafting the polymers on the silica surface, with high molecular weight and low grafting densities, without discernible differences in other material properties. In this context, Boday et al. ${ }^{14}$ grew the vinyl polymer from the silica surface by carrying out surface initiated atom transfer radical polymerization (SI-ATRP) of methyl methacrylate to the silica surface. This approach led to the incorporation of well-defined poly(methyl methacrylate) with low polydispersities and, therefore, allowed the establishment of tuneable structureproperty relationship between the molecular weight of the grafted polymer and toughening of the hybrid aerogel.

To date, controlled polymerization techniques have been scarcely investigated as an approach to reinforce silica aerogels' mechanical properties. There is no systematic report in the literature to investigate the mechanical reinforcement of silica aerogels by exploiting surface initiated reversible additionfragmentation chain transfer (SI-RAFT) ${ }^{\mathbf{1 5}, \mathbf{1 6}}$ polymerization technique. Inspired by a previous study ${ }^{\mathbf{1 4}}$ and in order to verify the effect of polymer molecular weight on the density and mechanical properties of aerogels, for the first time, we developed mechanically improved hybrid aerogels using SI-RAFT polymerization, and in this way well-defined polystyrene and poly(butyl acrylate) were grown from the silica surface. 
This work included the synthesis of sol-gel processable RAFT initiators, sol-gel polymerization for RAFT initiatormodified gels, RAFT from the surface of gels, supercritical drying for aerogel formation and characterization of the resulting aerogel composites. Not only will this approach allow for the incorporation of well-defined polymers into aerogels, but its versatile methodology allows for the polymerization of a number of vinyl and cross-linkable monomers, without the initiator causing weakening of the silica network. ${ }^{\mathbf{1 7}}$

\section{Experimental procedure}

\subsection{Materials}

Tetramethylorthosilicate (TMOS, 98\%), anhydrous methanol (99.5\%), toluene (99\%), tetrahydrofuran ( $\mathrm{THF}, \geq 99.9 \%$ ), 3(mercaptopropyl)trimethoxysilane (95\%), 1-propanethiol (98\%), carbon disulfide anhydrous (>99\%), benzyl chloride (99\%), sodium methoxide ( $25 \mathrm{wt} \%$ solution in methanol), butyl acrylate (BA, <99\%), styrene (St, <99\%), 2,2'-azobisisobutyronitrile (AIBN, 99\%) and ammonium hydroxide $\left(\mathrm{NH}_{4} \mathrm{OH}, 28-30 \mathrm{wt} \%\right.$ solution) were purchased from Sigma-Aldrich. All reagents were used without further purification.

\subsection{Preparation of S-Benzyl S'- trimethoxysilylpropyltrithiocarbonate (BTPT-silica) and S- benzyl $S^{\prime}$-propyltrithiocarbonate (BPTT)}

In a typical run, a solution of sodium methoxide in methanol ( $25 \mathrm{wt} \%, 6.48 \mathrm{~g}, 30 \mathrm{mmol}$ ) was added dropwise, under nitrogen, to a stirred solution of 3-(mercaptopropyl)trimethoxysilane $(95 \%, 6.20 \mathrm{~g}, 30 \mathrm{mmol})$ in $50 \mathrm{~mL}$ of anhydrous methanol. After stirring for $30 \mathrm{~min}, \mathrm{CS}_{2}(3.05 \mathrm{~g}, 40 \mathrm{mmol})$ was added dropwise to the solution, and the mixture was then stirred at ambient temperature for $5 \mathrm{~h}$. Benzyl chloride $(98 \%, 3.43 \mathrm{~mL}, 30 \mathrm{mmol})$ was added to the resultant yellow solution, and the mixture was stirred overnight under nitrogen. The mixture was concentrated, diluted with dichloromethane, filtered off, and concentrated under reduced pressure until constant weight. S-benzyl S'-trimethoxysilylpropyltrithiocarbonate (BTPT, $10.8 \mathrm{~g}, 30 \mathrm{mmol}, 102 \%$ ) was obtained as an orange oil and used without further purification.

S-benzyl S'-propyltrithiocarbonate (BPTT), another chain transfer agent (CTA), was synthesized according to a similar procedure but using 1-propanethiol as the raw material. BPTT was obtained as a yellow liquid product in $98.5 \%$ isolated yield.

The ${ }^{1}$ HNMR spectra of these two CTA are shown in Fig. S1 $\dagger$ (including the peak data), with their corresponding chemical structures.

BTPT. ${ }^{1} \mathrm{HNMR}\left(\mathrm{CDCl}_{3}\right): \delta 7.30(\mathrm{~m}, 5 \mathrm{H}, \mathrm{PhH}), 4.60\left(\mathrm{~s}, 2 \mathrm{H}, \mathrm{CH}_{2}\right)$, $3.56\left(\mathrm{~s}, 9 \mathrm{H}, \mathrm{CH}_{3} \mathrm{O}\right), 3.39$ (t, J7, 2H, $\left.\mathrm{CH}_{2} \mathrm{~S}\right), 1.84\left(\mathrm{~m}, 2 \mathrm{H}, \mathrm{CH}_{2}\right), 0.77$ $\left(\mathrm{t}, \mathrm{J} 8,2 \mathrm{H}, \mathrm{CH}_{2} \mathrm{Si}\right)(v d$. spectra a at Fig. $\mathrm{S} 1 \dagger)$.

BPTT. ${ }^{1} \mathrm{HNMR}\left(\mathrm{CDCl}_{3}\right): \delta 7.33(\mathrm{~m}, 5 \mathrm{H}, \mathrm{PhH}), 4.61(\mathrm{~s}, 2 \mathrm{H}$, $\mathrm{CH}_{2}$ ), 3.35 (t, J7, 2H, $\left.\mathrm{CH}_{2} \mathrm{~S}\right), 1.75\left(\mathrm{~m}, 2 \mathrm{H}, \mathrm{CH}_{2}\right), 1.02$ (t, J7, 3H, $\mathrm{CH}_{3}$ ) (vd. spectra b at Fig. $\left.\mathrm{S} 1 \dagger\right)$.

\subsection{Synthesis of RAFT modified silica (RAFT-Silica) aerogels}

A solution containing tetramethylorthosilicate (TMOS, $2.15 \mathrm{~mL}$, $14.7 \mathrm{mmol}$ ) and S-benzyl $\mathrm{S}^{\prime}$-trimethoxysilylpropyltrithiocarbonate (BTPT) $(0.1 \mathrm{~mL}, 0.3 \mathrm{mmol})$ was prepared. The second solution was prepared by adding $11.2 \mathrm{~mL}$ of the gelation solvent (methanol), $\mathrm{H}_{2} \mathrm{O}(1.08 \mathrm{~mL}, r=$ [water $\left.] /[\mathrm{Si}]=4\right)$ and $0.4 \mathrm{~mL}$ of $\mathrm{NH}_{4} \mathrm{OH}$ (28-30 wt\%). The two solutions were mixed for one minute, then poured in a poly(propylene) container, with a 17.2 $\mathrm{mm}$ nominal diameter, at room temperature. The final volume was $15 \mathrm{~mL}$. Gelation occurred within $\sim 5$ minutes. Gels were aged at room temperature for $48 \mathrm{~h}$ followed by $48 \mathrm{~h}$ at $50{ }^{\circ} \mathrm{C}$. Once removed from the poly(propylene) container, the methanol was slowly exchanged with toluene over $48 \mathrm{~h}$ for the RAFT process. If processed for silica aerogel formation, gels were introduced in the supercritical carbon dioxide $\left(\mathrm{scCO}_{2}\right)$ drying autoclave affording a RAFT-modified silica aerogel. For drying, the sample was first washed with methanol to ascertain the purification of monoliths and to increase the diffusivity of $\mathrm{scCO}_{2}$ on the wet gels. Unreacted silica precursors and organic residues were eliminated by passing the solvent through the monoliths for $1 \mathrm{~h}$ with a mass flow rate of $2 \mathrm{~mL} \mathrm{~min}^{-1}$ and a chamber pressure of 200 bar, under a constant temperature of $40{ }^{\circ} \mathrm{C}$. Then, the samples were exposed to the flow of supercritical $\mathrm{CO}_{2}$ for $90 \mathrm{~min}$, having a mass flow rate of $5 \mathrm{~mL} \mathrm{~min}{ }^{-1}$.

\subsection{RAFT polymerizations}

Initiator-modified gels were placed into a Schlenk flask along with toluene $(50 \mathrm{~mL})$. The Schlenk vial was sealed and purged with argon for 30 minutes. In a separate vial, the monomer (BA or $\mathrm{St}$ ) and the radical initiator AIBN were deoxygenated by bubbling nitrogen for 30 minutes. Then, the deoxygenated monomer ( $45 \mathrm{mmol}$ ) and AIBN ( $0.1 \mathrm{~g})$ were transferred into the Schlenk flask (under argon) containing the gel in toluene, using a syringe.

The solution was allowed to equilibrate for $3 \mathrm{~h}$, after which the Schlenk flask was placed into a thermostatic oil bath at $70{ }^{\circ} \mathrm{C}$. The time left in the oil bath, i.e. the polymerization time, depended upon the desired composite density. After a specific reaction time, the polymer-silica composite aerogels were removed from the vial and washed several times with toluene in order to purify the composite gels from the free polymer and other residues. Afterward, the toluene solution was slowly exchanged for methanol, which is needed for supercritical drying.

\subsection{General procedure to cleave grafted chains from the silica surface by aminolysis}

The grafted polymers were cleaved from the silica surface according to a method similar to the literature. ${ }^{12}$ In a typical run, the following compounds were added to a glass tube: 100 $\mathrm{mg}$ of polymer-silica aerogels, $5.0 \mathrm{~mL}$ of THF, and one drop of a diluted aqueous solution of $\mathrm{Na}_{2} \mathrm{~S}_{2} \mathrm{O}_{4}$. The solution was degassed with nitrogen for 5-10 $\mathrm{min}$, and then $0.1 \mathrm{~mL}$ of degassed $n$-hexylamine was injected into the mixture. After stirring at ambient temperature overnight, the solution was subjected to 
centrifugation and the recovered polymer was used for sizeexclusion chromatography (SEC) analysis.

\subsection{Characterization techniques}

Molecular weights of the PBA and PSt grafted from the surface of silica aerogels were determined by high-performance sizeexclusion chromatography (HPSEC), using a ViscotekTDAmax, with a differential viscometer (DV), a right-angle laser-light scattering (RALLS, Viscotek), a low-angle laser-light scattering (LALLS, Viscotek) and refractive index (RI) detectors. The column set consisted of a PL $10 \mathrm{~mm}$ guard column $(50 \times 7.5$ $\mathrm{mm}^{2}$ ), followed by one Viscotek T200 column $(6 \mathrm{~mm})$, one MIXED-EPL gel column $(3 \mathrm{~mm})$ and one MIXED-C PL gel column $(5 \mathrm{~mm})$. A HPLC dual piston pump was set with a flow

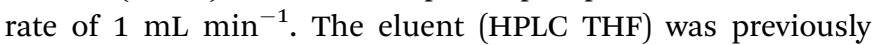
filtered through a $0.2 \mathrm{~mm}$ filter. The system was also equipped with an on-line degasser. The tests were conducted at $30{ }^{\circ} \mathrm{C}$ using an Elder $\mathrm{CH}-150$ heater. Before injection $(100 \mu \mathrm{L})$, the samples were filtered through a poly(tetrafluoroethylene) (PTFE) membrane with $0.2 \mu \mathrm{m}$ pore. For PBA and PSt, $M_{\mathrm{n}, \mathrm{GPC}}$ and PDI values were determined by conventional calibration with PSt standards between 1820 and 96000.

$400 \mathrm{MHz}{ }^{1} \mathrm{H}$ nuclear magnetic resonance (NMR) spectra of samples of the reaction mixture were recorded on a Bruker Avance III $400 \mathrm{MHz}$ spectrometer, with a $5 \mathrm{~mm}$ TXI triple resonance detection probe, in $\mathrm{CDCl}_{3}$ with tetramethylsilane (TMS) as an internal standard.

Infrared analysis was conducted with ATR-FTIR spectroscopy (JASCO FTIR-4100). Elemental analysis was performed using ET 1108 CHNS-O (FISIONS Instruments), detection limits: $\mathrm{N}=$ $0.25 \%, \mathrm{C}=35 \%, \mathrm{H}=0.15 \%, \mathrm{O}=0.25 \%, \mathrm{~S}=0.35 \%$. The bulk density $\left(\rho_{\mathrm{b}}\right)$ was determined by measuring the weight and volume of the samples. Dimensional shrinkage (\%) was taken as the difference between the diameters of the aerogel monolith and of the $20 \mathrm{~mL}$ syringe mold (nominally $17.2 \mathrm{~mm}$ ). A He pycnometer (Accupyc 1330, Micromeritics) was used to measure the real (skeleton) density of the samples. By combining the information of the skeleton and bulk densities, it was possible to evaluate the porosity of the samples. In addition, we used the nitrogen gas adsorption technique (Accelerated Surface Area and Porosimetry ASAP 2000, Micromeritics) for the determination of the specific surface area, pore size distribution, pore surface area and pore volume of the materials. Before the analysis, the sample was outgassed at $60{ }^{\circ} \mathrm{C}$ in vacuum $\left(10^{-5}\right.$ bar) for $24 \mathrm{~h}$, to remove the adsorbed species. In the analysis, volumes of the adsorbed nitrogen at five different relative pressures $(0.05$ to 0.2$)$ were taken at $77 \mathrm{~K}$, in order to obtain the specific surface area by the BET theory. The desorption isotherm and the $\mathrm{BJH}$ theory were used for the porosimetry evaluation.

Thermal gravimetric analysis (TGA) was performed using a TA model TGA-Q500 instrument with a heating rate of $10{ }^{\circ} \mathrm{C}$ $\mathrm{min}^{-1}$, from room temperature to $600{ }^{\circ} \mathrm{C}$, under a nitrogen atmosphere.

A scanning electron microscope (SEM) (JMS-5310, JOEL) was used to observe the material microstructure. Due to the low electrical conductivity of the highly porous silica-based samples, an Au film was deposited on their surface, using the Physical Vapour Deposition (PVD) technique for $20 \mathrm{~s}$.

The thermal conductivity of the reinforced aerogels was measured using a transient method (Thermal constants analyzer TPS $2500 \mathrm{~S}$, Hot Disk). The sensor is clamped between two identical disc shaped pieces of the sample, which have a diameter of $1 \mathrm{~cm}$ and thickness of $0.5 \mathrm{~cm}$ (properly cut from the cylindrical aerogel samples). This analysis was carried out at $20{ }^{\circ} \mathrm{C}$ and the equipment presents a reproducibility and accuracy over $1 \%$ and $5 \%$, respectively.

For the mechanical test, samples were cut with a length: diameter ratio of $2: 1$, and were polished to make sure that the top and bottom sides were smooth and parallel. The compression test was conducted following the ASTM standard D695-02a. All tests were carried out under nominal room conditions with a stroke speed of $1.3 \mathrm{~mm} \mathrm{~min}^{-1}$.

\section{Results and discussion}

A typical synthesis route towards SI-RAFT is illustrated in Fig. 1. First, the trimethoxysilane-containing chain transfer agent Sbenzyl $\mathrm{S}^{\prime}$-trimethoxysilylpropyltrithiocarbonate (BTPT-silica) was synthesized as a RAFT-silane coupling agent. ${ }^{15}$ Then, modified silica wet gels (RAFT-silica) were synthesised by conducting the sol-gel polymerization reaction between TMOS and BTPT-silica. The lower reactivity of the trimethoxysilyl group compared to TMOS during the sol-gel reaction ensured that the majority of initiating groups would be inserted to the aerogel surface. ${ }^{18}$ The RAFT polymerization process initiated from the chain transfer agent (CTA) surface-modified silica, and, finally, the resultant aerogel composites were supercritically dried. The degree of polymerization was $[M]_{\mathrm{o}} /[I]_{\mathrm{o}}=150$. Polymerization times were varied from 6 to $30 \mathrm{~h}$, thus allowing for control over the grafted polymers' molecular weight (Table 1).

SI-RAFT of vinyl monomers was conducted in solution using AIBN and BPTT as a free CTA in the presence of a monolithic gel. The monolithic gels were subjected to several solvent exchanges with the initiators and free CTA at room temperature before heating to start the polymerizations. For surface RAFT polymerization, the addition of free RAFT-CTA is an useful

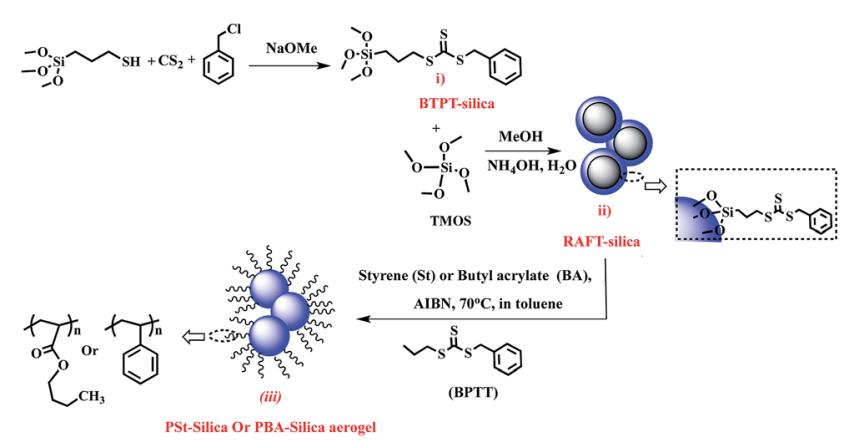

Fig. 1 Preparation of initiator modified gel and RAFT growth of PSt and PBA on the silica surface. 
Table 1 Properties of native silica, PBA- and PSt-silica aerogel composites

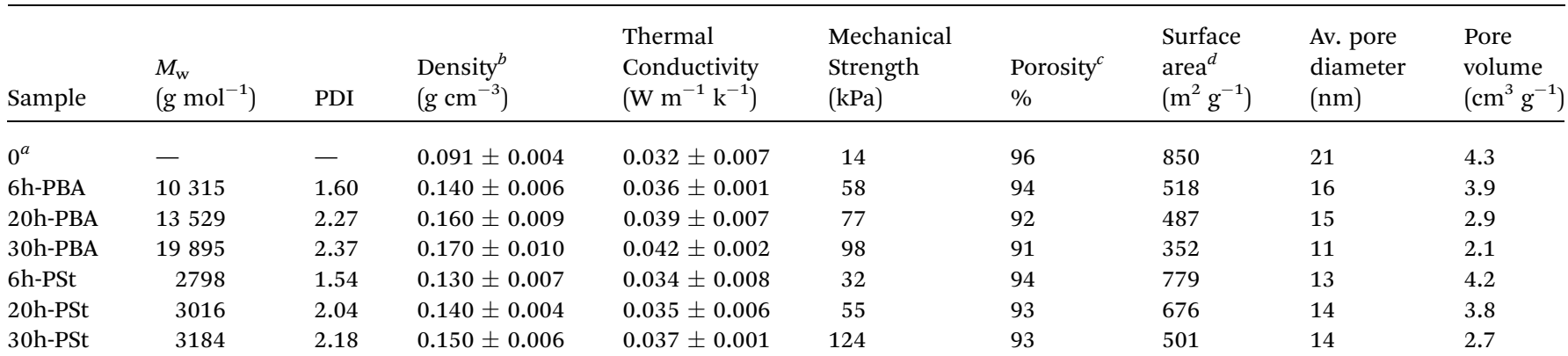

${ }^{a}$ Silica aerogel prepared without PBA/PSt (RAFT-silica). ${ }^{b}$ Density values are an average from three prepared gels. ${ }^{c}$ Porosity calculated from $\left[\left(\frac{1}{\rho_{\mathrm{b}}}-\frac{1}{\rho_{\mathrm{s}}}\right) / \frac{1}{\rho_{\mathrm{s}}}\right] \times 100 \%, \rho_{\mathrm{b}}=$ bulk density $\rho_{\mathrm{s}}=$ skeletal density. ${ }^{d}$ Surface area calculated from the $\mathrm{N}_{2}$ adsorption isotherm using the BET method.

approach to help control the polymerization process when the concentration of surface-bound RAFT-CTA is insufficient. ${ }^{19}$

Imaging the PBA and PSt silica aerogel composite using SEM (Fig. 2b and c) revealed that the composites' aggregate structure had been thickened compared to that of the polymer free RAFT initiator silica aerogel (Fig. 2a). Thickening of the aerogels' aggregate structure was expected from the grafted PSt and PBA, due to a reinforcement of the weak necks in aerogels' aggregate structure.

TGA, FT-IR and elemental analysis (EA) confirmed that chain transfer agents were successfully immobilized on the silica surface. The elemental analysis revealed that loading of BTPTsilica (CTA agents) was $0.017 \mathrm{mmol}$ of CTA per gram of solid. In the FT-IR spectrum of the plain BTPT-silica aerogel sample (spectrum a of Fig. 3), a strong and broad absorption band corresponding to the asymmetric stretching vibration of $\mathrm{Si}-\mathrm{O}-$
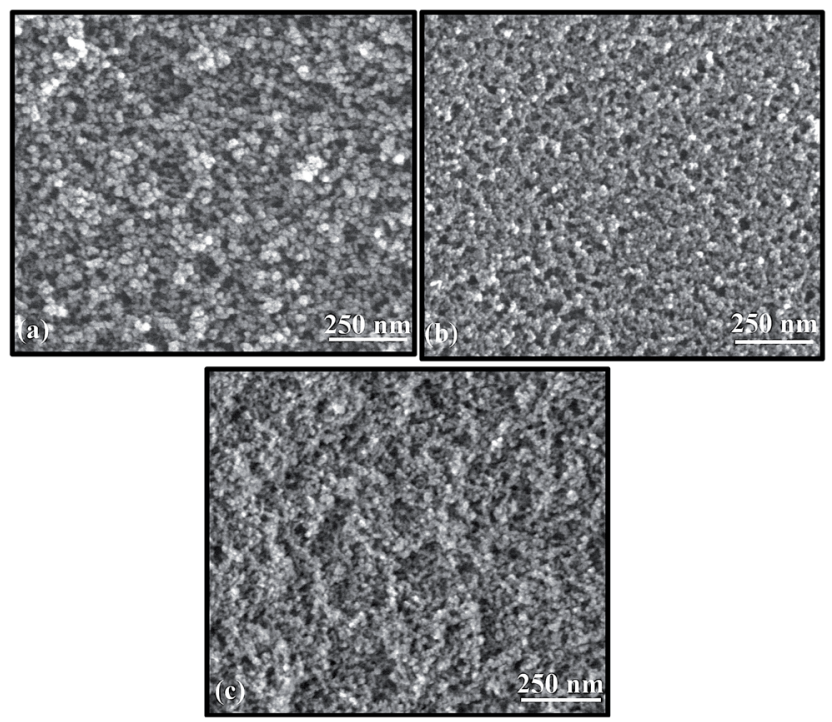

Fig. 2 (a) SEM image of surface-functionalized silica aerogel (RAFTsilica), (b) PBA-silica aerogel and (c) PSt-silica aerogel after $30 \mathrm{~h}$ of polymerization.
Si was noted at $1020-1050 \mathrm{~cm}^{-1}$ and characteristic absorption bands of aromatic rings were observed at 1464 and $1616 \mathrm{~cm}^{-1}$. The characteristic absorption bands of $\mathrm{C}=\mathrm{O}$ stretching were observed at $1727 \mathrm{~cm}^{-1}$ in the PBA-silica aerogel (spectrum b of Fig. 3); the characteristic peaks of PSt-silica appeared at 1452, 1492 and $1601 \mathrm{~cm}^{-1}$ (spectrum c of Fig. 3).

Determination of tethered PSt and PBA molar mass was achieved by detaching the polymer from $\mathrm{SiO}_{2}$ by aminolysis using $n$-hexylamine ${ }^{\mathbf{1 5}}$ and size exclusion chromatography (SEC) of recovered polymers. Controlled SI-RAFT was achieved, as evidenced by SEC of cleaved PBA in the range of $M_{\mathrm{w}}=10-20 \mathrm{~kg}$ $\mathrm{mol}^{-1}$ and PSt in the range of $M_{\mathrm{w}}=2-3 \mathrm{~kg} \mathrm{~mol}^{-1}$, with polydispersities $\left(M_{\mathrm{w}} / M_{\mathrm{n}}\right)$ of 1.6-2.4 and 1.5-2.1, respectively - Fig. 4a and b. Clearly, the molecular weight of PSt and PBA increased with the polymerization time, thus providing strong evidence for controlled growth of the polymer. PDI, which was initially quite low, became moderate and then even quite high at high polymer loadings. These molecular weight distributions were

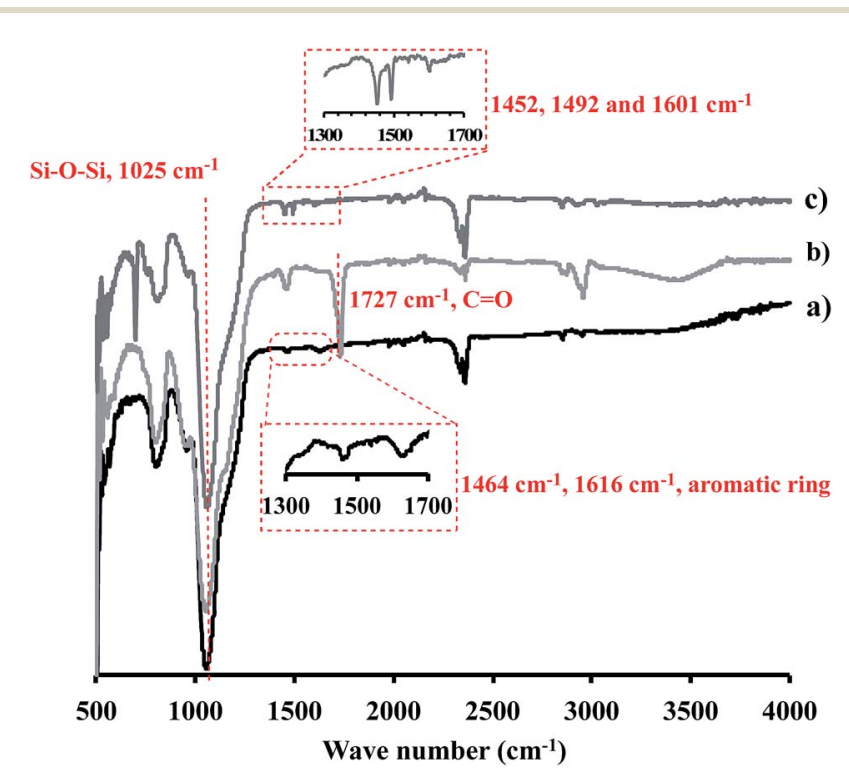

Fig. 3 FT-IR spectra of (a) RAFT-silica, (b) PBA-silica and (c) PSt-silica. 
a)

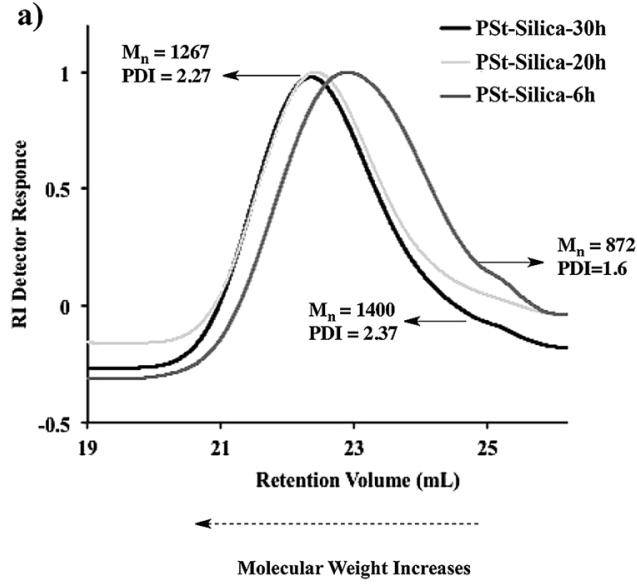

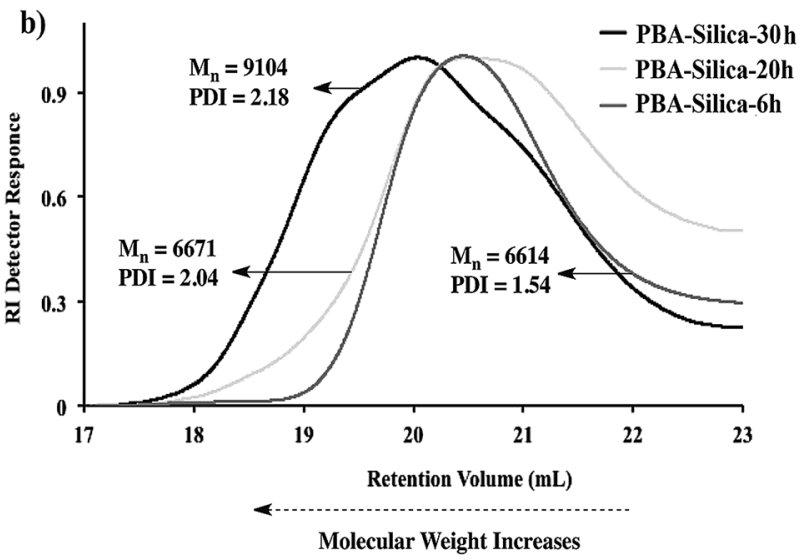

Fig. 4 Size exclusion chromatography traces for (a) PSt and (b) PBA polymers isolated from silica composites.

broader than those observed for typical homogeneous free polymer in solution.

It is very common that the polydispersity of grafted polymers from highly porous materials is higher than those prepared in the absence of a substrate. Typically it ranges from 1.14 to 2.59 , which were found to be higher with increased molecular weight. Moreover, the complex environment of aerogels and the concave surface geometry lead to sterically inaccessible initiation sites that affect the overall control of the SI-RAFT. ${ }^{20}$

The thermal stability and grafting ratio of the polymer on the silica surface were studied by thermogravimetric analysis (TGA). The increase in the molecular weight of the polymer was correlated with the content of the polymer in PSt-silica or PBAsilica composites - Table S1. $\dagger$ Fig. S2 $\uparrow$ indicates that the onset temperature of non-reinforced silica aerogels increased by the incorporation of the polymer on the silica surface. This result also suggests that the thermal stability of silica composites increased by increasing the grafting ratio of the composites.

On the basis of SEC, TGA and gas adsorption data, the initiator efficiency, that is, the ratio of the number of grafted polymer chains to the number of initiation sites has been determined and ranged between 1.5 and $24 \%$ - Table S1. $\dagger$ The apparent decrease in the grafting density and initiation efficiency of the PBA in the composites may be related to the coupling of chains at the later stages of the grafting process, when the pores are nearly completely filled with the polymer and the monomer cannot readily diffuse to convert the propagating chains to dormant species. ${ }^{20}$ This observation is consistent with the more than two-fold increase in the molecular weight and the broadening of the molecular weight distribution at this stage. This broadening in the PDI of the grafted polymers can also be explained by comparing the composition/organic content of outer and inner sides of PBA-silica aerogels prepared in $20 \mathrm{~h}$ and $30 \mathrm{~h}$ of polymerization (Fig. 5). After thirty hours of RAFT, the difference in the organic content between the center and exterior part has increased to $10 \%$. While the growth of polymer chains inside the pores was hindered because of the scarcity of available space, the chains growing on the external surface had no such restriction. Therefore, as the

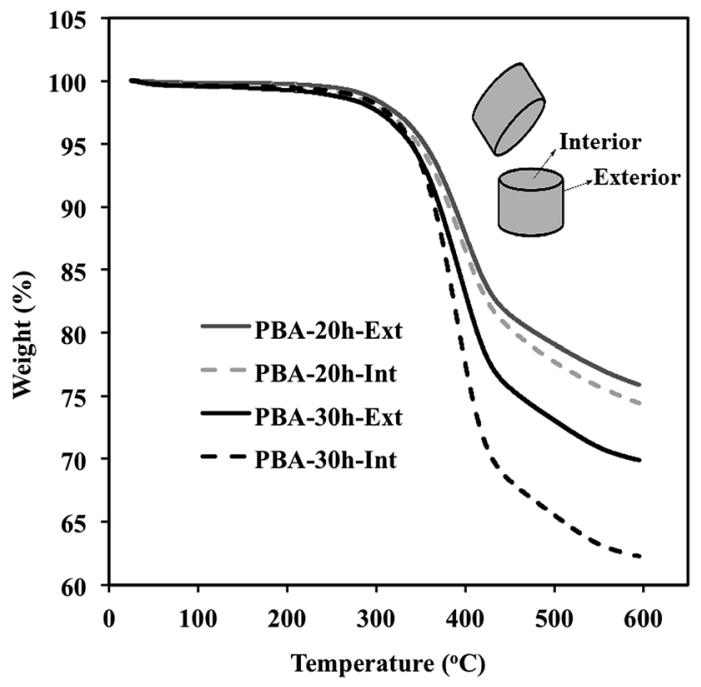

Fig. 5 TGA analysis of samples from interior and exterior of silica aerogels RAFT modified with PBA for 20 and 30 hours.

polymerization proceeds, it causes second molecular weight distribution and the increase in the grafted polymers' PDI.

The porous nature of aerogel composites was evaluated by nitrogen physisorption measurements (Fig. 6a and b). The detailed results are summarized in Table 1 . It was found that the isotherms of almost all the samples were of type IV, according to the IUPAC classification, which is the characteristic of mesopore structures with cylindrical pores. The specific surface areas and the pore size distributions were evaluated from the adsorption and desorption branches of the isotherms applying the Brunauer-Emmett-Teller (BET) and Barrett-Joyner-Halenda (BJH) methods, respectively (Fig. $6 \mathrm{c}$ and d). The nitrogen adsorption of silica composites decreases gradually as the polymerization time increases. Grafting of PSt (Fig. 6a) and PBA (Fig. 6b) resulted in a gradual decrease in the volume of nitrogen adsorbed as the density of the composites increases. The volume of the adsorbed gas decreases due to the expected gradual filling of the mesopores within the silica aerogels RAFT- 

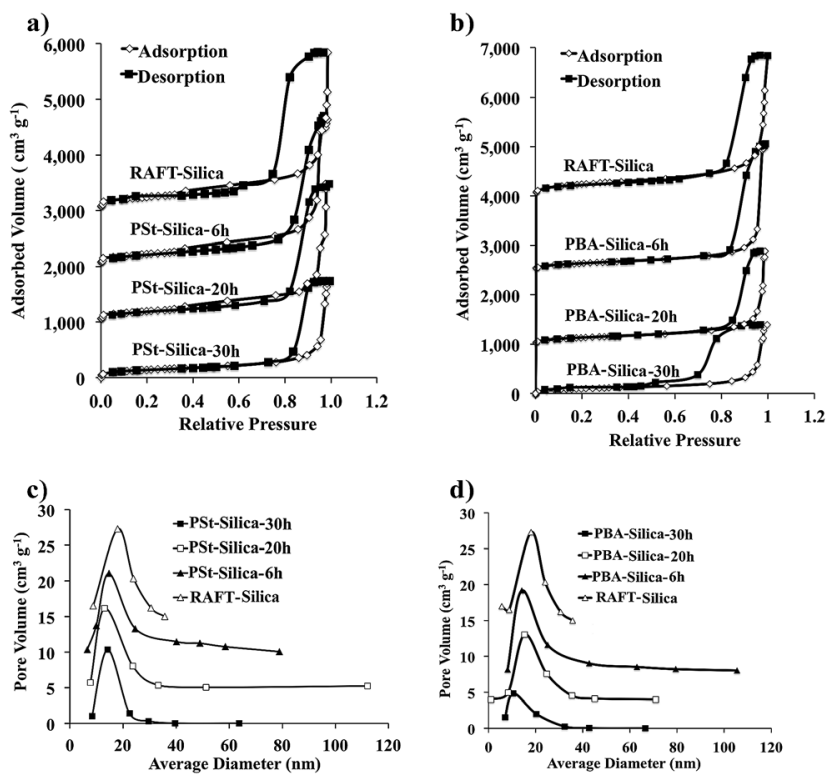

Fig. 6 ( $a$ and b) Nitrogen adsorption and desorption isotherms and (c, d) Barrett-Joyner-Halenda (BJH-desorption) pore-size distributions of aerogels. Relative pressure $=P / P_{0}$.

modified with the polymer. As a consequence, the average pore size shifts to lower values as the density of composites (PStSilica and PBA-silica) increases.

The thermal conductivity of the synthesised aerogels has been measured by a non-steady state (transient) approach and the results for nonreinforced and reinforced aerogels are indicated in Table 1 and Fig. 7. The thermal conductivity of aerogels strongly depends on the number of network connecting points and on the applied mass to reinforce them; it ranges from 0.036 to $0.042 \mathrm{~W} \mathrm{~m}^{-1} \mathrm{~K}^{-1}$ for PBA-reinforced aerogels and from 0.034 to $0.037 \mathrm{~W} \mathrm{~m}^{-1} \mathrm{~K}^{-1}$ for PSt-reinforced aerogels. The thermal conductivity of reinforced aerogels slightly increases by increasing the molecular weight of the grafted polymer and the density of aerogel composites. However, for PSt-reinforced aerogels, due to the low molecular weight of the grafted polymer compared to the grafted PBA, the increase in the thermal conductivity is less pronounced.

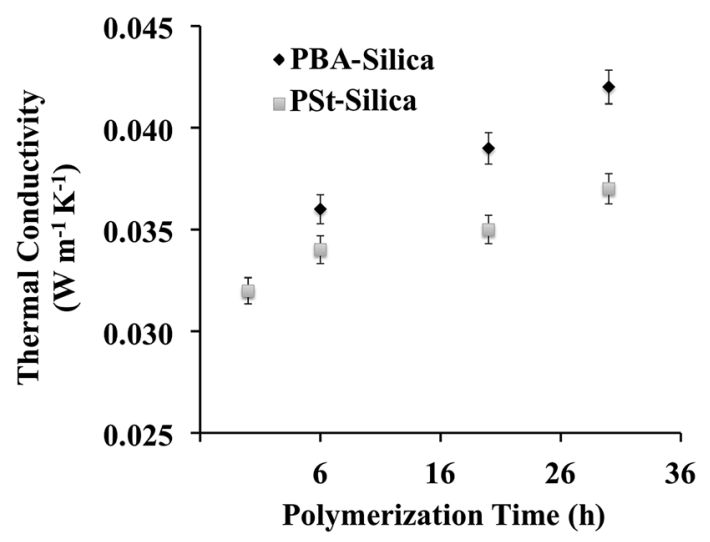

Fig. 7 Thermal conductivity versus polymerization time.
The effects of grafting PSt and PBA to strengthen aerogels are evaluated from sample stress-strain curves shown in Fig. 8a. The values of the compression strength (maximum) obtained from these curves are summarized in Table 1. The RAFT-silica aerogel, which is modified by using the initiator (no PSt or PBA), with an average density of $0.091 \mathrm{~g} \mathrm{~cm}^{-3}$, is slightly weaker than pure silica aerogels (max. strength $\sim 30 \mathrm{kPa}$ ) with the same density. The reduction in strength is observed in other silica aerogels synthesized by other co-monomers of trialkoxysilanes. ${ }^{14}$ The strength of the PBA and PSt-silica aerogel composites increases with the density of the composite aerogels and the molecular weight of the polymers. The sample photographs at Fig. 8b show that reinforced aerogels have enough structural integrality and mechanical strength compared to the native TMOS based and RAFT modified aerogels.

The brittle behavior of the silica aerogel remains after the introduction of the RAFT initiator. However, even a low polymer content promotes a clear improvement of the mechanical properties of the material: the Young's modulus increases and the compression strength becomes 5-9 times higher. Furthermore, the mechanical behavior improvement is noticeable from the much higher energy that the hybrid aerogel is able to absorb up to the maximum compression strength (roughly the area under the stress-strain curve).

The initiator-modified aerogels are transparent and less dense; the transparency somehow is retained for PBA-reinforced aerogels. The PSt-silica aerogel looks opaque and stronger than RAFT-silica and PBA-silica aerogels.

a)

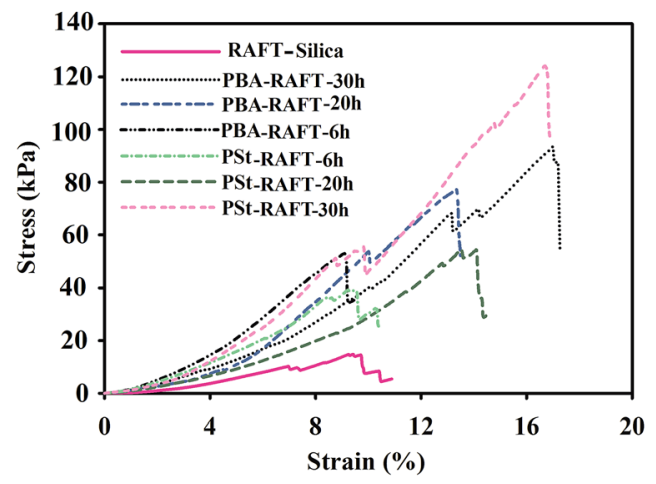

b)

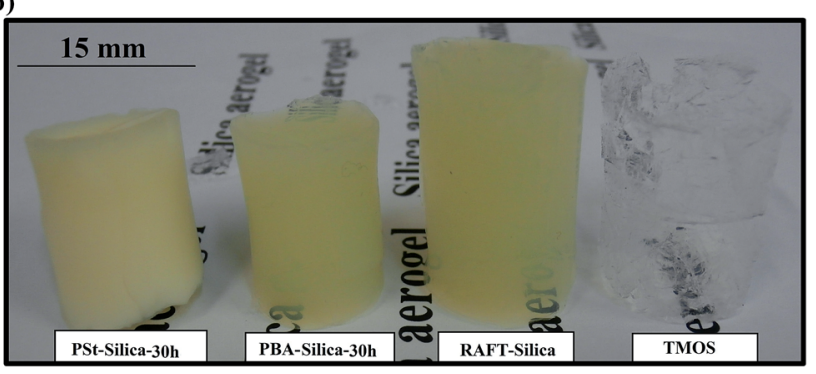

Fig. 8 (a) Stress strain curves of different reinforced and nonreinforced aerogels; (b) photograph of plain TMOS-derived aerogel and initiator-modified aerogel (RAFT-silica), along with reinforced PBA-silica and PSt-silica aerogels with 30 hours of polymerization time. 
The PBA-silica aerogel composite prepared with a polymerization time of $30 \mathrm{~h}$ and with a grafted molecular weight of $\sim 20 \mathrm{~kg} \mathrm{~mol}^{-1}$ increased the compression strength to $98 \mathrm{kPa}$. This is approximately $7 \times$ stronger than the initiator-modified aerogels with less than $2 \times$ increases in the density $\left(\rho_{\mathrm{b}}=0.17 \mathrm{~g} \mathrm{~cm}^{-3}\right)$. The strongest PSt-silica aerogel composite had a density of $0.15 \mathrm{~g} \mathrm{~cm}^{-3}$, a polymer molecular weight $\left(M_{\mathrm{w}}\right)$ of $3 \mathrm{~kg} \mathrm{~mol}^{-1}$ and a compression strength of $124 \mathrm{kPa}$. This is $9 \times$ stronger than the initiator-modified silica aerogel. As indicated in Fig. 8a, despite the low molecular weight of PSt compared to PBA, the PStreinforced aerogels were at the same time stronger, tougher and stiffer than PBA-reinforced aerogels for all the polymerization times. For the same polymerization time and the same density, these aerogels were even stronger than PMMA-reinforced aerogels $(48 \mathrm{kPa})$ with a higher $M_{\mathrm{w}}\left(63 \mathrm{~kg} \mathrm{~mol}{ }^{-1}\right) .{ }^{14}$

Therefore, from these results, it can be concluded that the molecular weight of the grafted polymer plays an important role in the composites' mechanical properties and, depending on the type and nature of the growing polymers, different results can be achieved for mechanical strengthening of aerogels.

\section{Conclusions}

In summary, we have demonstrated the controlled growth of grafted polymers from the surface of a silica gel, by implementing the RAFT polymerization technique for the first time. The polydispersities were in the range of 1.5-2.4. We established a relationship between the main properties (bulk density, mechanical properties and thermal insulation) of silica aerogel composites and the molecular weight of polymers used to reinforce the composite. It was found that, as the molecular weight increases, so does the composite mechanical properties, but many of the aerogel physical properties are retained. The approach described herein for the synthesis of polymer-silica composite aerogels promises to be applicable to a wide variety of polymers that can be polymerized in a controlled way via RAFT.

\section{Acknowledgements}

The research leading to these results has received funding from the European Union Seventh Framework Programme (FP7-MCITN) under grant agreement no. 264710. The authors would like to thank the Directorate-General for Science, Research and
Development of the European Commission for financial support of the research.

\section{Notes and references}

1 J. P. d. Cunha, F. Neves and M. I. Lopes, Nucl. Instrum. Methods Phys. Res., Sect. A, 2000, 452, 401-421.

2 P. Tsou, J. Non-Cryst. Solids, 1995, 186, 415-427.

3 P. Tsou, D. E. Brownlee, S. A. Sandford, F. Horz and M. E. Zolensky, J. Geophys. Res.: Planets, 2003, 108, 81138134.

4 L. W. Hrubesh, J. Non-Cryst. Solids, 1998, 225, 335-342.

5 L. W. Hrubesh, Mater. Res. Soc. Symp. Proc., 1995, 381, 267.

6 N. Leventis, Acc. Chem. Res., 2007, 40, 874-884.

7 H. Maleki, L. Durães and A. Portugal, J. Non-Cryst. Solids, 2014, 385, 55-74.

8 J. P. Randall, M. A. B. Meador and S. C. Jana, ACS Appl. Mater. Interfaces, 2011, 3, 613-626.

9 S. Yun, H. Luo and Y. Gao, J. Mater. Chem. A, 2014, 2, 1454214549.

10 D. J. Boday, R. J. Stover, B. Muriithi, M. W. Keller, J. T. Wertz, K. A. D. Obrey and D. A. Loy, Chem. Mater., 2008, 20, 28452847.

11 D. J. Boday, R. J. Stover, B. Muriithi, M. W. Keller, J. T. Wertz, K. A. D. Obrey and D. A. Loy, ACS Appl. Mater. Interfaces, 2009, 1, 1364-1369.

12 H. Maleki, L. Durães and A. Portugal, Microporous Mesoporous Mater., 2014, 197, 116-129.

13 N. Leventis, C. Sotiriou-Leventis, G. Zhang and A.-M. M. Rawashdeh, Nano Lett., 2002, 2, 957-960.

14 D. J. Boday, P. Y. Keng, B. Muriithi, J. Pyun and D. A. Loy, J. Mater. Chem., 2010, 20, 6863-6865.

15 Y. Zhao and S. Perrier, Macromolecules, 2007, 40, 9116-9124. 16 J. Moraes, K. Ohno, T. Maschmeyer and S. Perrier, Chem. Commun., 2013, 49, 9077-9088.

17 S. Mulik, C. Sotiriou-Leventis, G. Churu, H. Lu and N. Leventis, Chem. Mater., 2008, 20, 5035-5046.

18 B. Radhakrishnan, A. N. Constable and W. J. Brittain, Macromol. Rapid Commun., 2008, 29, 1828-1833.

19 Z. Qu, F. Hu, K. Chen, Z. Duan, H. Gu and H. Xu, J. Colloid Interface Sci., 2013, 398, 82-87.

20 M. Kruk, B. Dufour, E. B. Celer, T. Kowalewski, M. Jaroniec and K. Matyjaszewski, Macromolecules, 2008, 41, 8584-8591. 